\title{
Rolle der Zytologie in der hämatopathologischen Diagnostik
}

Bode, Beata ; Tinguely, M

\begin{abstract}
The role of cytology has so far been underrecognized in the diagnostic process of hematopathological questions. This article presents an algorithm which allows a stepwise work-up of cytology specimens obtained by minimally invasive ultrasound-guided fine needle aspiration in patients with unexplained lymph node swelling. Moreover, it is shown how the selective separation of cytology specimens allows the application of immunophenotypic analysis including flow cytometry and immunohistochemistry as well as molecular analyses, such as fluorescence in situ hybridization (FISH) and polymerase chain reaction (PCR) strategies. With the integrative procedure presented, cytology offers an excellent cost-effective tool for the diagnostic approach of patients with suspected hematopathological malignancies allowing a high diagnostic accuracy, ideal for initial diagnosis or follow-up.
\end{abstract}

DOI: https://doi.org/10.1007/s00292-012-1580-1

Other titles: Role of cytology in hematopathological diagnostics

Posted at the Zurich Open Repository and Archive, University of Zurich

ZORA URL: https://doi.org/10.5167/uzh-65159

Journal Article

Published Version

Originally published at:

Bode, Beata; Tinguely, M (2012). Rolle der Zytologie in der hämatopathologischen Diagnostik. Der Pathologe, 33(4):316-323.

DOI: https://doi.org/10.1007/s00292-012-1580-1 
Pathologe 2012 $\cdot 33: 316-323$

DOI 10.1007/s00292-012-1580-1

Online publiziert: 10. Juni 2012

๑) Springer-Verlag 2012

Schwerpunktherausgebe

M. Tötsch, Graz
B. Bode $\cdot$ M. Tinguely

Institut für Klinische Pathologie, Universtitätsspital Zürich, Schweiz

\section{Rolle der Zytologie in der hämatopathologischen Diagnostik}

Die aktuelle Klassifikation der Weltgesundheitsorganisation (WHO) 2008 zu Tumoren des hämatopoietischen und lymphatischen Systems sieht einen integrativen Diagnoseansatz vor. Darunter versteht sie die Einbeziehung morphologischer, immunphänotypischer und molekularer Veränderungen einer Neoplasie wie auch der klinischen Befunde. Sie bezweckt damit, einen Tumor einer umschriebenen Entität zuzuordnen sowie prognostische und prädiktive Veränderungen als Grundlage für Therapieentscheidungen einzubinden. Der Zytologie, als Spezialgebiet der Pathologie, wurde bisher eine untergeordnete Rolle in der hämatopathologischen Diagnostik zuerkannt. Wir beschreiben hier neue Möglichkeiten, Vorteile und Grenzen.

Die aktuelle WHO-Klassifikation 2008 der Tumoren des hämatopoietischen und lymphatischen Systems [21] basiert auf einem integrativen Konzept. Dieses schließt morphologische, immunphänotypische und molekulare Aberrationen eines Tumors ebenso wie klinische Befunde ein. Um diesen Anforderungen an eine moderne Diagnostik gerecht zu werden, braucht es zum einen eine erweiterte technische Ausstattung, aber ebenso einen gebietsübergreifenden Austausch an Fachkompetenz. Im diagnostischen Prozess zu berücksichtigen ist eine patienten- sowie kostenorientierte Haltung. Die Zytologie als eine minimalinvasive Methode ist in vielen klinischen Situationen bestens ge- eignet für die rasche Abklärung unklarer Lymphadenopathien [2, 4, 12, 17, 24, 27].

Die Zytologie wird in der Schweiz mit einer einjährigen Zusatzausbildung nach dem Facharzt für Pathologie als eigenständiges Fach mit einem Zusatztitel von der schweizerischen Ärztekammer (FMH) anerkannt. Am Institut für Pathologie des Universitätsspitals Zürich wird bereits seit mehr als 30 Jahren erfolgreich in der Abteilung für Zytologie ein Ambulatorium betrieben ( $\bullet$ Abb. 1a), in welchem Zytopathologen selber (nach Bedarf auch ultraschallgesteuerte) Feinnadelpunktionen (FNP) oberflächlicher Organe vornehmen (Lymphknoten, Schilddrüse, Brust, Weichteile, Haut).

Die Ultraschallsteuerung (• Abb. 1b) hat sich zwischenzeitlich als äußerst hilfreiche Methode erwiesen, um zielsicher, qualitativ hochwertiges zytologisches Material auch aus nicht palpablen Knoten (z. B. aufgrund Positronenemissionstomographie, PET, erhobene Befunde) zu gewinnen [13, 23]. Ein Leistungsnachweis in Sonographie ist neben weiteren Fachrichtungen auch den Zytopathologen in einer Zusatzausbildung der Schweizerischen Gesellschaft für Ultraschall in der Medizin (SGUM) zugänglich - analog zur
Abb. 1 a Ambulatorium der Abteilung für Zytologie, Institut für Klinische Pathologie, Universitätsspital Zürich. b Die Ultraschallsteuerung der Feinnadelpunktion wird zur Bestätigung der korrekten Platzierung der Nadel innerhalb der Zielstruktur (Pfeile) sowie der Vermeidung einer akzidentellen Verletzung benachbarter Strukturen in 70-80\% der Eingriffe benutzt

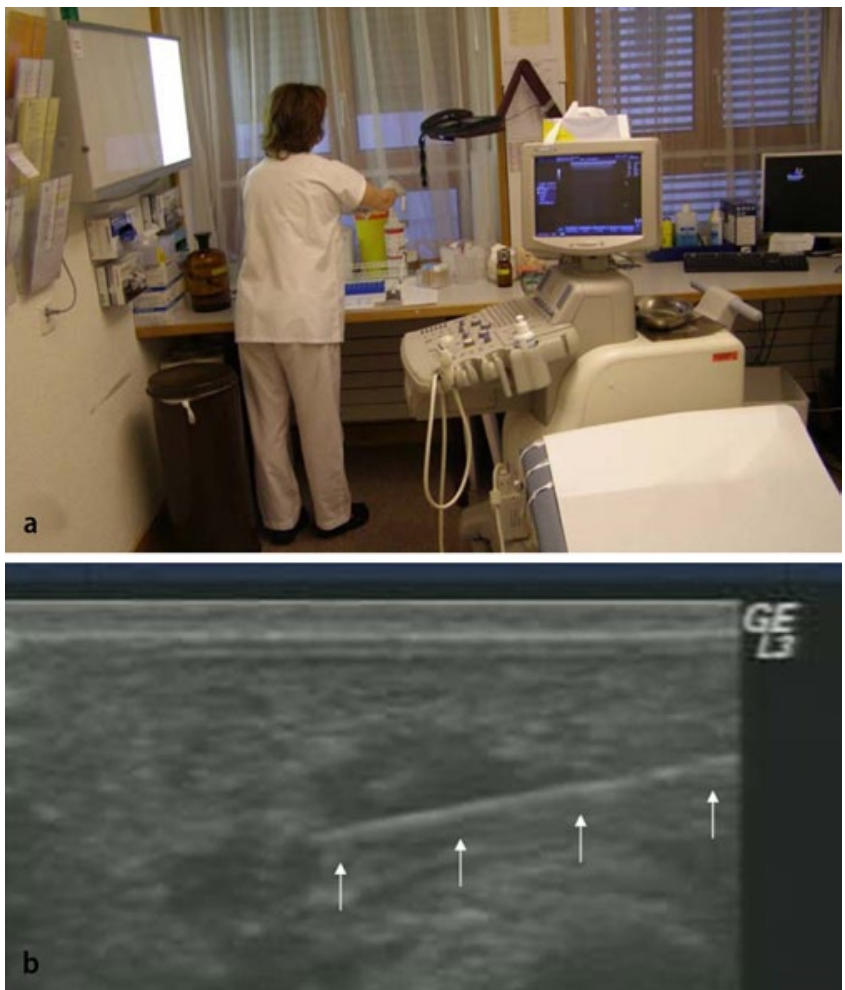




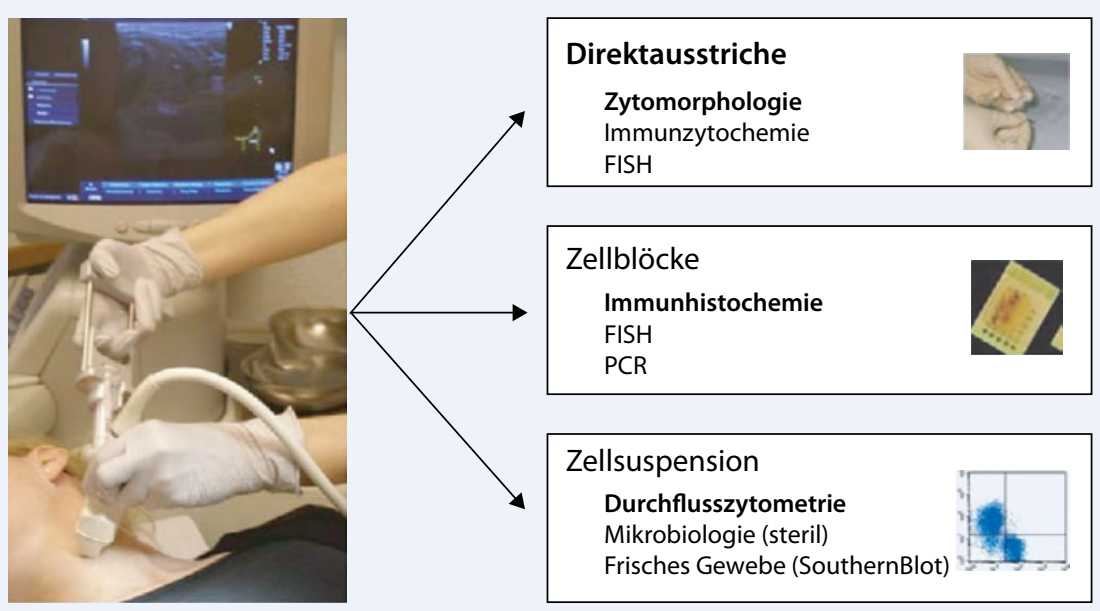

Abb. $2 \Delta$ Die mithilfe einer Feinnadelpunktion eines Lymphknotens gewonnene Probe wird nach obligater Anfertigung von 1 bis 3 Direktausstrichen in Abhängigkeit von klinischer Situation und Fragestellung für weitere Zusatzuntersuchungen gezielt aufgeteilt. FISH Fluorescence in situ hybridization", $P C R$ Polymerasekettenreaktion

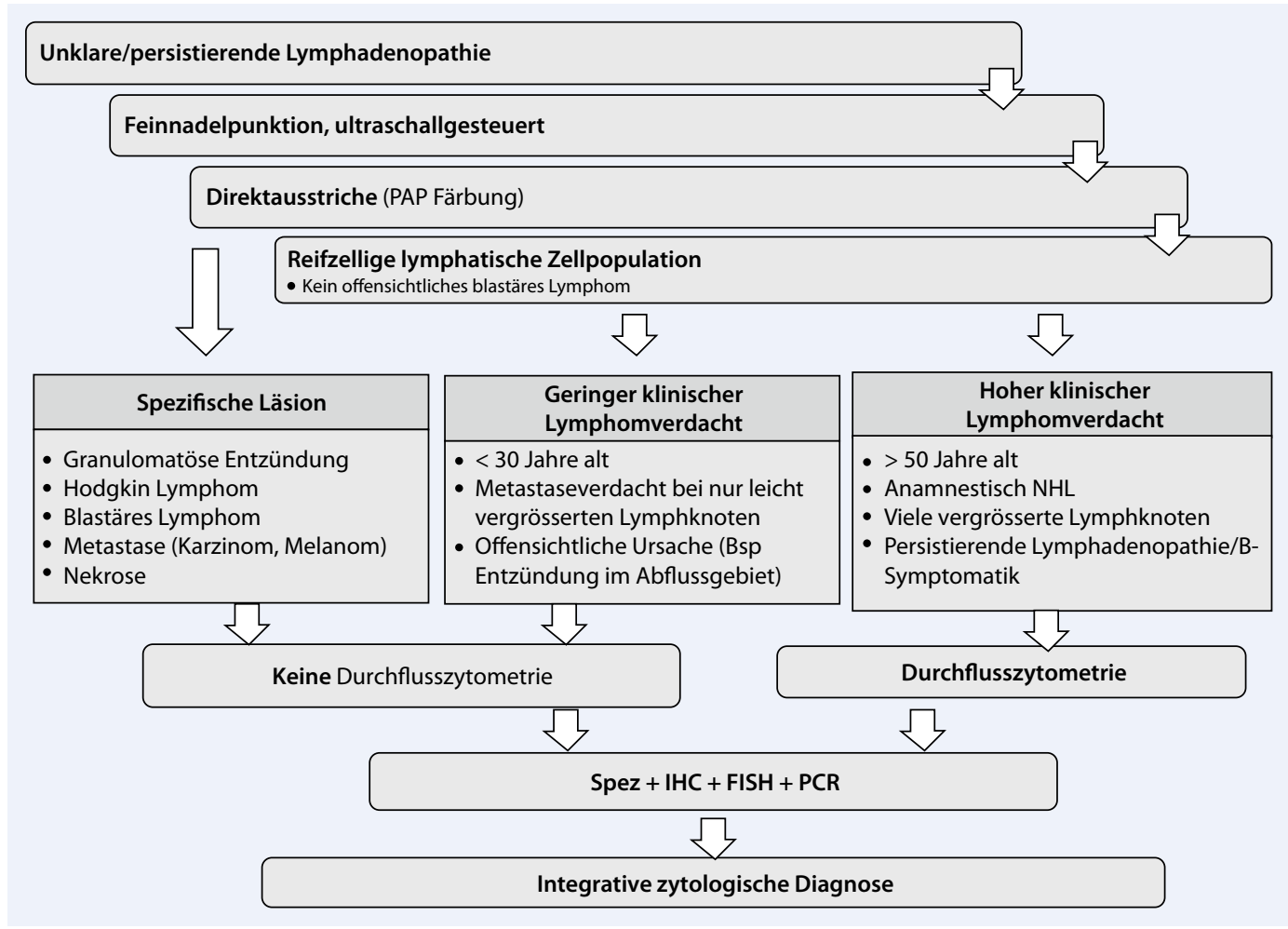

Abb. $3<$ Algorithmus für die integrative schrittweise zytologische Diagnostik von Lymphadenopathien. Zentral ist die Beurteilung der Direktausstriche (innerhalb $1 \mathrm{~h}$ vorliegend). Weiterführende Zusatzuntersuchungen werden aufgrund der jeweiligen individuellen klinischen Situation veranlasst, was besonders wichtig ist für 30- bis 50-jährige Patienten. Spez Gram-, PAS-, Grocott-, und/ oder Ziehl-Neelsen-Färbungen; IHC Immunhistochemie; $F I S H$, fluorescence in situ hybridization", PCR Polymerasekettenreaktion

Deutschen Gesellschaft für Ultraschall in der Medizin (DGUM) und zur Österreichischen Gesellschaft für Ultraschall in der Medizin (ÖGUM).

Pro Jahr beurteilen wir FNP von durchschnittlich 2300 Lymphknoten. Beinahe die Hälfte (45\%) der FNP werden im Ambulatorium der Abteilung für Zytologie von erfahrenen Zytopathologen durchgeführt. In 20-25\% der FNP der Lymphknoten sowie in $5-10 \%$ der zu untersuchen- den Flüssigkeiten werden wir mit hämatopathologischen Fragestellungen konfrontiert (Rezidive und Primärdiagnosen hämatologischer Erkrankungen). Mithilfe von umfangreichen Zusatzuntersuchungen, wie der Immunhistochemie an Zellblöcken, Durchflusszytometrie, „fluorescence in situ hybridization” (FISH), Polymerasekettenreaktion (PCR; • Abb. 2), werden die Proben als reaktiv, maligne bzw. unklarer Diqnität klassifiziert. Die
Biopsierate beträgt für Lymphknoten, die als reaktiv diagnostiziert wurden, $15 \%$ und bei malignen bzw. unklaren Befunden 4050\%. Die anschließende Biopsierate liegt, insbesondere im Rahmen der Primärdiagnostik, deutlich höher (80-90\%). Insbesondere bei reifzelligen Lymphomen und Anwendung moderner diagnostischer Methoden erreichen Sensitivität und Spezifität hinsichtlich der Lymphomdiagnose jeweils über $80 \%$ [1, 2, 8, 9, 14, 15, 19, 26]. 
Wir erläutern im Folgenden unseren algorithmisch aufgebauten diagnostischen Prozess der klinisch unklaren Lymphadenopathien (• Abb. 3) und werden den Schwerpunkt auf die ultraschallgesteuerte Feinnadelpunktion legen sowie den integrativen Ansatz in Sinne der geltenden WHO-Klassifikation. Wir gehen auf die Vorteile und Grenzen dieser Methode in der hämatopathologischen Diagnostik ein.

\section{Ultraschallgesteuerte Feinnadelpunktion}

Die durch den Zytopathologen selbst vorgenommene ultraschallgesteuerte Feinnadelpunktion im eigenen Ambulatorium (• Abb. 1) hat folgende entscheidende Vorteile:

1. Anamnese und klinische Befunde können direkt vom Patienten erhoben werden.

2. Die Sonographie erlaubt eine Beurteilung der Form der Lymphknoten und deren topographischer Verhältnisse (- Abb. 1b, Abb. 4a, Abb. 5a, Abb. 6a, Abb. 7a),

3. Die Punktion ist für den Patienten minimalinvasiv und komplikationsarm. Die Patienten können durch die Polikliniken/Hausärzte direkt im Anschluss an die Sprechstunde zugewiesen werden (keine Wartezeiten; Zeitaufwand für Patienten 20-30 min).

4. Der die FNP durchführende Zytopathologe bürgt für die Qualität der entnommenen Probe und triagiert das gewonnene Material in Abhängigkeit von der jeweils vorliegenden klinischen Situation für die notwendigen Zusatzuntersuchungen ( $\bullet$ Abb. 2).

\section{3-Säulen-Modell der zytologischen Lymphknotendignostik}

\section{Feinnadelpunktion und Materialaufarbeitung}

Das Aspirat wird (in Abhängigkeit von der Größe und Lage des Knotens, meist unter sonographischer Kontrolle, • Abb. 1b) durch fächerförmige Punktion mittels einer in einem Griff (z. B. Cameco ${ }^{\odot}$ ) montierten, 10-ml-Spritze mit konventioneller
Nadel (24G) entnommen. In den meisten Fällen sind 2 bis höchsten 3 aufeinanderfolgende Punktionen ausreichend. Das gewonnene Aspirat wird anschließend in mindestens 3 Portionen aufgeteilt (• Abb. 2):

1. alkoholfixierte, nach Papanicolaou gefärbte 1-3 Direktausstriche,

2. mit Cyto Lyte (Hologic Inc., Lausanne, Schweiz) versehene Zellsuspension zur Anfertigung eines formalinfixierten, in Paraffin eingebetteten Zellblockes und

3. in PBS entnommene Zellsuspension.

Je nach gewonnenem Material können unterschiedliche Analyseverfahren Anwendung finden.

Für die Veranlassung der Zusatzuntersuchungen entscheidend ist die morphologische Beurteilung der Direktausstriche (- Abb. 3), welche bereits innerhalb von 60 min zur Einsicht vorliegen. Die Papanicolaou-Färbung (• Abb. 4b, Abb. 5b, Abb. 6b, Abb. 8a) zeigt sich in unseren Händen als ideale Methode zur Beurteilung der Zellmorphologie, die es ferner zulässt, nachträglich ohne aufwändige Spezialvorbehandlung immunzytochemische ( $\bullet$ Abb. 7c) und/oder FISH-Untersuchungen (- Abb. 7e) daran durchzuführen (entscheidender Vorteil gegenüber den luftgetrockneten, MGG-gefärbten Ausstrichen).

Geleitet von der Morphologie am Ausstrich (insbesondere nach Ausschluss spezifischer entzündlicher Prozesse, Metastasen, blastärer und Hodgkin-Lymphome), kann eine zusätzliche Durchflusszytometrie (FACS) an der Zellsuspension in PBS durchgeführt werden (Ergebnis ist spätesens innerhalb von $24 \mathrm{~h}$ vorliegend; - Abb. 6c, Abb. 7b). Der Zellblock hat den Nachteil, dass die Morphologie durch die Zentrifugation der Zellen im Vergleich zum Ausstrich etwas eingeschränkt beurteilbar ist. Er birgt aber den Vorteil, dass je nach Materialmenge nicht nur isolierte Zellen, sondern sogar kleinste Gewebefragmente (• Abb. 5c) aspiriert werden, was eine Mitbeurteilung der Mikroumgebung von Tumorzellen erlaubt. Analog zur Gewebeeinbettung lassen sich am Zellblock an zahlreichen Schnittpräparaten immunhistochemische Zusatzfärbungen durchführen (• Abb. 5c, Abb. 6c), die
Pathologe 2012 $\cdot 33: 316-323$

DOI 10.1007/s00292-012-1580-1

(c) Springer-Verlag 2012

\section{B. Bode $\cdot$ M. Tinguely \\ Rolle der Zytologie in der hämatopathologischen Diagnostik}

\section{Zusammenfassung}

Die Zytologie stellt ein wichtiges diagnostisches Instrument in der täglichen Praxis der Hämatopathologie dar. Durch den zytologisch erfolgten Nachweis nichthämatologischer Ursachen einer Knotenbildung (z. B. Speicheldrüsenneoplasien, Tuberkulose, Melanom- oder Karzinommetastasen) besteht seltener die Notwendigkeit einer chirurgischen Exzision. Die kombinierte Anwendung morphologischer, immunphänotypischer sowie molekularer Analysen und der fachliche Austausch machen diese Untersuchungstechnik sicher. Somit stellt die zytologische Untersuchung in verschiedensten Situationen eine Alternative zur Exzisionsbiopsie dar - auch bei primären hämatologischen Erkrankungen und insbesondere bei Rezidiven.

\section{Schlüsselwörter}

Zytologie · Hämatopathologie .

Feinnadelpunktion - Lymphom .

Durchflusszytometrie

\section{Role of cytology in hematopathological diagnostics}

\section{Abstract}

The role of cytology has so far been underrecognized in the diagnostic process of hematopathological questions. This article presents an algorithm which allows a stepwise workup of cytology specimens obtained by minimally invasive ultrasound-guided fine needle aspiration in patients with unexplained lymph node swelling. Moreover, it is shown how the selective separation of cytology specimens allows the application of immunophenotypic analysis including flow cytometry and immunohistochemistry as well as molecular analyses, such as fluorescence in situ hybridization (FISH) and polymerase chain reaction $(P C R)$ strategies. With the integrative procedure presented, cytology offers an excellent cost-effective tool for the diagnostic approach of patients with suspected hematopathological malignancies allowing a high diagnostic accuracy, ideal for initial diagnosis or follow-up.

\section{Keywords}

Cytology · Hematopathology · Fine needle aspiration - Lymphoma · Flow cytometry 

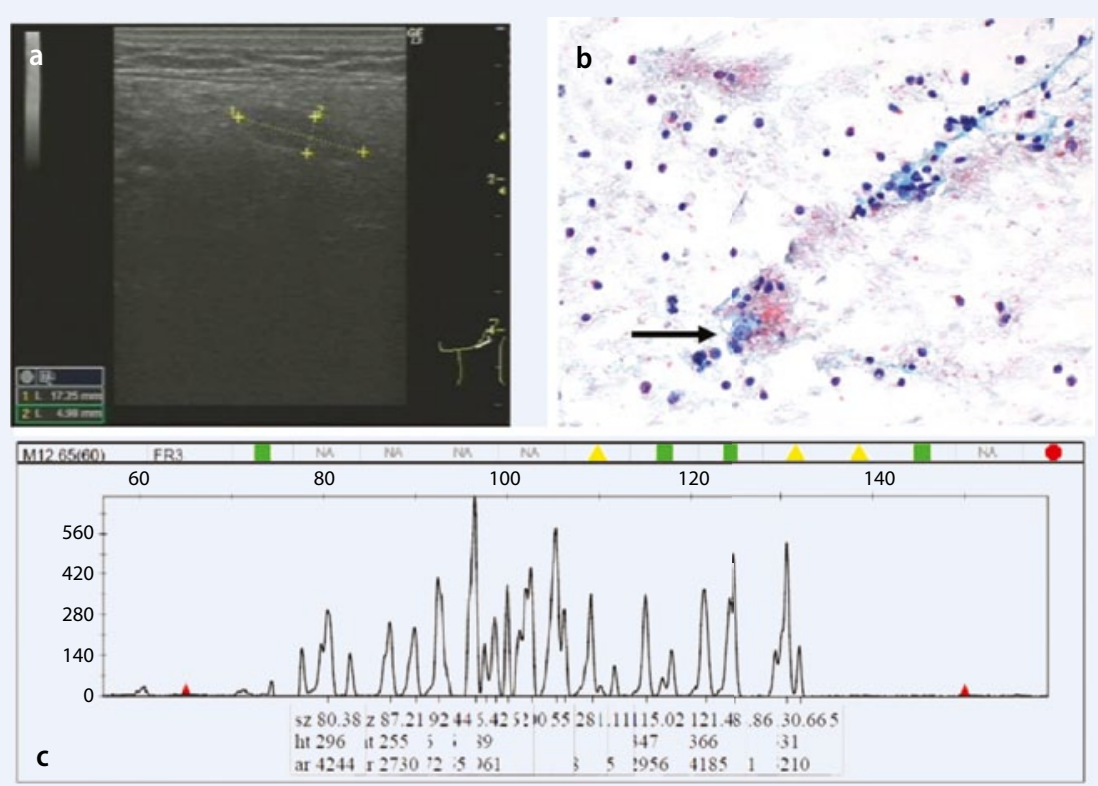

Abb. $4 \Delta$ Bei einer 46-jährigen Frau mit der Vorgeschichte eines diffus großzelligen Lymphoms wurde im Verlauf ein in einer PET-CT-Untersuchung schwach positiver Lymphknoten submandibulär festgestellt. a Die Ultraschalluntersuchung wies einen ovalären, gering vergrößerten Lymphknoten nach. b Die ultraschallgesteuerte Feinnadelpunktion erbrachte eine ausschließlich gemischte Population von Lymphozyten mit einzelnen Kerntrümmermakrophagen (Pfeil, Direktausstrich, Papanicolaou-Färbung; Vergr. 200-fach). c Die molekulargenetische Klonalitätsanalyse zeigte ein polyklonales Rearrangierungsmuster für die Immunglobulinschwerkette. Diagnose: reaktiv veränderter Lymphknoten
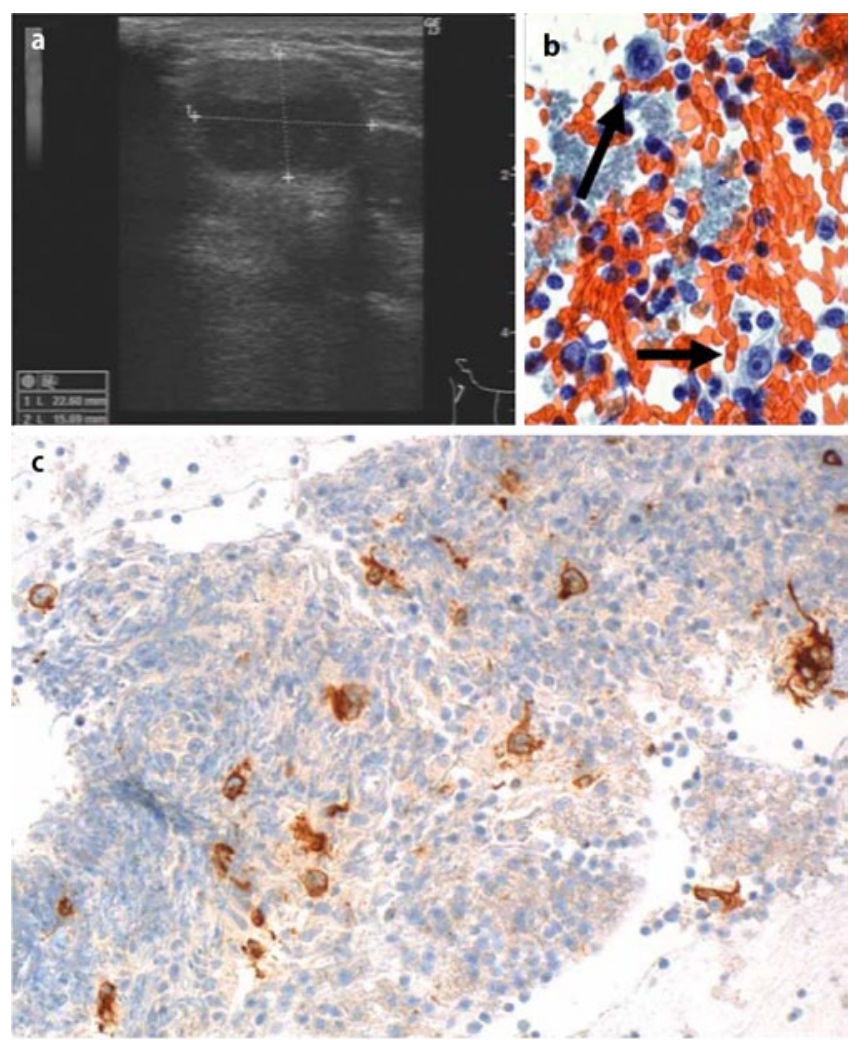

Abb. $5<$ a Persistierende Lymphadenopathie zervikal rechts bei einer 40-jährigen Frau. Die 2 Jahre zuvor stattgehabte histologische Untersuchung eines Lymphknotens ergab einen Normalbefund. $\boldsymbol{b}$ In den Direktausstrichen der Feinnadelpunktion fanden sich einige Hodgkin-Zellen (Pfeile) in Begleitung unauffälliger Lymphozyten (Papanicolaou-Färbung; Vergr. 400-fach). c Die Immunhistochemie auf den Mikrostanzen im Zellblock zeigte mehrere CD30-positive Zellen (Vergr. 200-fach) mit schwacher Koexpression von PAX5. Diagnose: Hodgkin-Lymphom mit identischen Färbeprotokollen wie das restliche Einsendegut gefärbt werden. Außerdem lassen sich auch hier In-situ-Hybridisierungen durchführen, die entweder mit chromogenen ( $\bullet$ Abb. 7d) oder fluoreszenzmarkierten Farbstoffen gefärbt werden können. RNA oder DNA kann sowohl von frischer Zellsuspension wie auch vom Zellblock gewonnen und für weitere molekulare Analysen mittels PCR eingesetzt werden ( $\bullet$ Abb. 4c, Abb. 8b).

\section{Durchflusszytometrie}

Die Durchflusszytometrie (FACS) (- Abb. 6c, Abb. 7b) liefert innerhalb von wenigen Stunden Informationen über gleichzeitig mehrere Marker an der Oberfläche der untersuchten Zellen. Die Stärke der Durchflusszytometrie zeigt sich vor allem in der Unterscheidung von reaktiven lymphoproliferativen Läsionen vs. niedrig maligner Non-Hodgkin-Lymphome, besonders der B-Zell Reihe sowie deren Klassifikation [1, 2, 7, 8, 11, 19, 27]. Die Durchflusszytometrie kommt deshalb im diagnostischen Prozess nach klinischer, makroskopischer (sonographischer) und morphologischer Abklärung im Rahmen eines Algorithmus zum Zuge (• Abb. 3).

Wir verwenden dazu ein beschränktes Set an Oberflächenmarkern für BLymphozyten (CD19, CD20; CD5, CD10, CD23) sowie T-Lymphozyten (CD3, CD4, CD8; CD2, CD5). Bei eindeutig blastären, vor allem großzelligen Lymphomen verzichten wir meistens bewusst auf eine FACS-Untersuchung, da hier aufgrund der Fragilität des Zytoplasmas der neoplastische Zellen die Aussage oft eingeschränkt ist und das immunhistochemische Markerset, das im Zellblock zur Anwendung kommt, größer ist und den diagnostischen Anforderungen entspricht.

\section{Molekulare Analysen}

\section{Interphasenfluoreszenz- in-situ-Hybridisierung}

Zytologiematerial, besonders Ausstrichpräparate sind prädestiniert für zytogenetische Untersuchungen, insbesondere Interphasen-FISH-Untersuchungen (- Abb. 7e; $[3,5,6,10,16,18,28]$ ). 

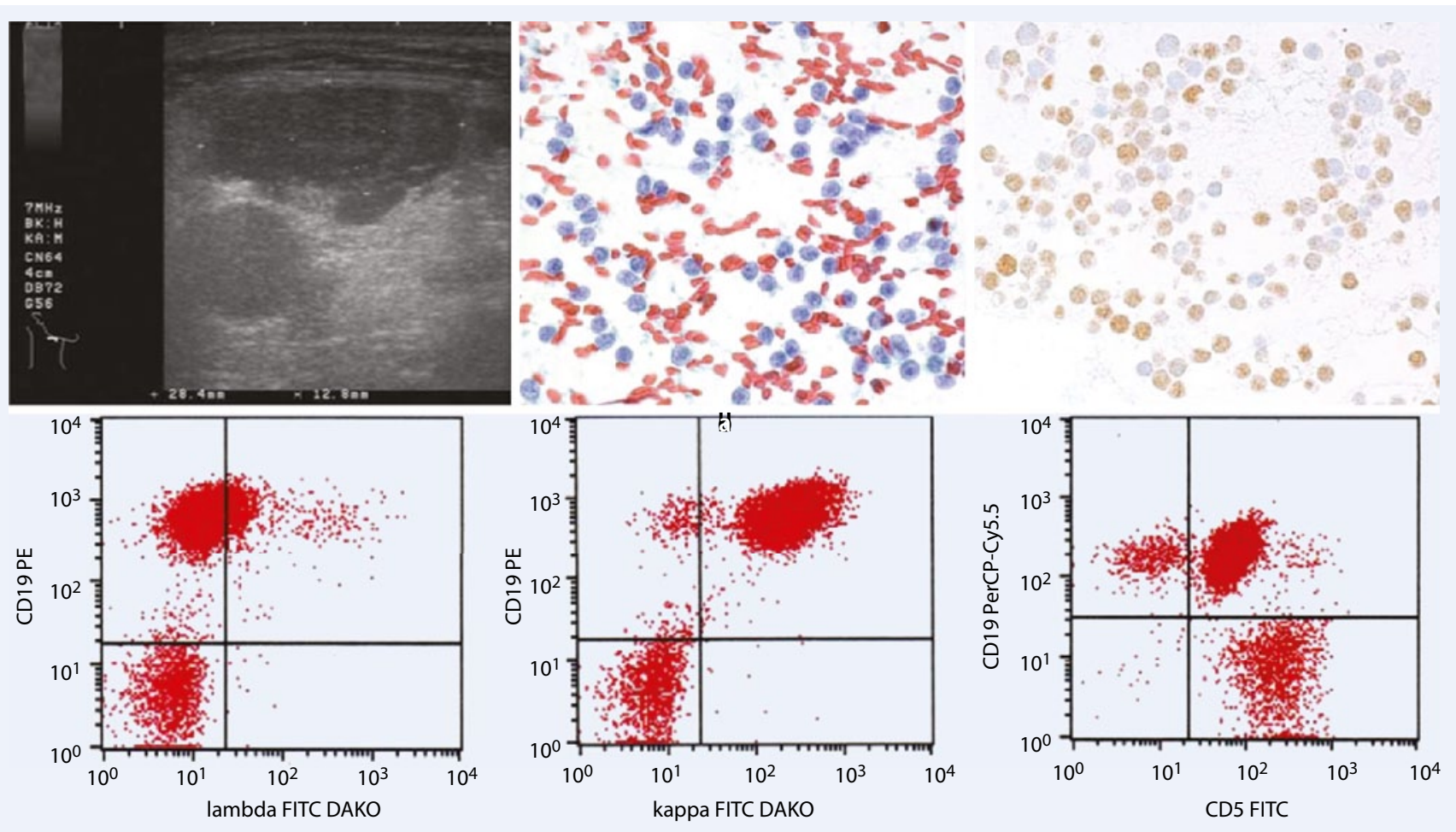

Abb. $6 \Delta$ Bei einer 64-jährigen Patientin mit bekanntem Kolonkarzinom wurden klinisch vergrößerte rechtsseitige, submandibuläre Lymphknoten festgestellt, welche sonographisch pathologische Form zeigten (a). b Die Feinnadelpunktion ergab eine monomorphe Population lymphatischer Zellen ohne Nachweis von Karzinomzellen (Direktausstrich, Papanicolaou-Färbung;Vergr. 400-fach). c Die Durchflusszytometrie zeigte eine kappa-Leichtkette-restringierte B-Zell-Population, welche CD5 koexprimiert und eine nukleäre Positivität für Bcl1 zeigt d (Zellblock; Vergr. 400-fach)

Hierzu eignen sich nach unserer Erfahrung PAP-gefärbte, aber auch native Ausstriche. Sie haben den Vorteil, dass schnittbedingte Artefakte wie Überlagerungen und Eigenfluoreszenz durch Bindegewebe und somit die Rate an falsch positiven Signalen reduziert werden kann. Die Ausstrichpräparate eignen sich deshalb besonders für Untersuchungen mit der Frage eines Chromosomenoder Genlokusverlustes, da diese Untersuchungen einer besonders hohen falsch positiven Rate unterliegen. Diese beträgt am Ausstrich etwa 5-20\% und am Gewebe sogar bis zu $50 \%$.

Die optische Tiefenschärfe der FISHSignale kann durch sog. Z-Stapel-Aufnahmen am Mikroskop und durch Herausfiltern von Streulicht unterschiedlicher Fokusebenen mittels eines in den Strahlengang gelegten Gitters (ApoTome, Carl Zeiss AG, Feldbach, Schweiz) noch zusätzlich verbessert werden. Letzteres ist von besonderem Vorteil bei dickeren, aus Zellblöcken gewonnenen Schnittpräparaten.
Im zytologischen Untersuchungsgut für hämatopathologische Fragestellungen wie hier beschrieben kommen v. a. lokusspezifische Proben zur Anwendung. Diese können benutzt werden, um numerische Aberrationen wie Vorhandensein oder Fehlen spezifischer Sequenzen (z. B. des p53) zu detektieren.

Am meisten Anwendung auf diesem Gebiet finden jedoch Proben zur Detektion von strukturellen Aberrationen, also Translokationen (- Abb. 7e). Hier empfiehlt sich, ein minimales Set an „Breakapart"-Sonden wie BCL-1, BCL-2, BCL-6, $\mathrm{C}-\mathrm{MYC}$ anzubieten. Zentromer-Proben eignen sich vor allem für den Nachweis von Aneuploidien, da sie an chromosomenspezifischen, repetitiven Sequenzen hybridisieren.

Für jede Probe muss eigens eine falsch positive Rate, ein sog. „cut-off” erstellt werden. Die falsch positive Rate bezieht sich auf abnormale Signalmuster im Normalgewebe und wird meist als Mittel abnormer Signale mit 3-facher Standardabweichung berechnet. Werte, die also über dem „cut-off” liegen, gelten als echte molekulare Veränderungen.

\section{Polymerasekettenreaktion und "southern blot"}

Als Ausgangsmaterial für PCR-Untersuchungen kommt meist der Zellblock zum Zuge (• Abb.4c), seltener Flüssigkeiten (• Abb. 8b) oder frisches Zellaspirat. Lezteres eignet sich vor allem für Klonalitätsanalysen mittels „southern blot“, vorausgesetzt, die Zellmenge ist ausreichend.

Für die Zellblockpräparate gelten die gleichen Einschränkungen wie für das übliche in Paraffin eingebettete Gewebe. Es kann sowohl DNA wie RNA gewonnen werden. Anwendungen finden meist Klonalitätsuntersuchungen der schweren Kette des Immunglobulins (- Abb. 4c) und des T-Zell-Rezeptors (• Abb. 8b), Mutationsanalysen der schweren Kette des Immunglobulins (bspw. als Prognosefaktor bei chronisch lymphozytischem Lymphom/B-CLL), seltener Analysen für Translokationen $\mathrm{t}(14 ; 18)$ oder $\mathrm{t}(11 ; 14)$. 

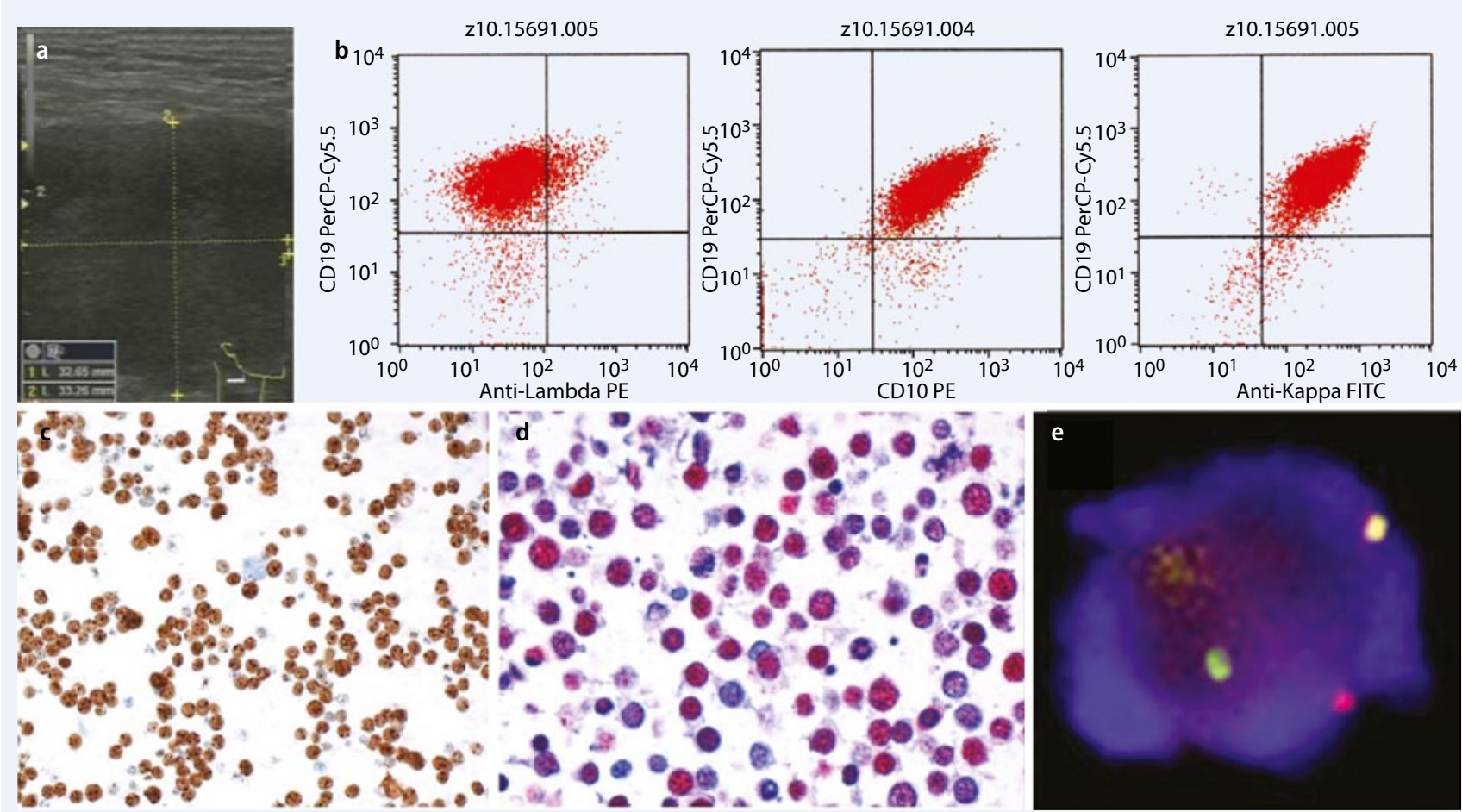

Abb. $7 \Delta$ Ein bis dahin gesunder 38-jähriger Mann stellte einen schnell wachsenden, rechtsseitigen Knoten am Hals fest, sonographisch $>3 \mathrm{~cm}$ groß. a Die mittels Feinnadelpunktion gewonnene lymphatische Zellpopulation zeigte eine kappaLeichtketten-Restriktion der CD19-positiven B-Zellen (b). c Die Immunzytochemie für MIB1 auf dem Direktausstrich zeigte einen Proliferationsindex von >95\% (Vergr. 200-fach). d Die chromogene In-situ-Hybridisierung für EBER erbrachte den positiven Epstein-Barr-Virus-Nachweis (Zellblock; rotes Reaktionsprodukt; Vergr. 630-fach). e Die auf dem Direktausstrich durchgeführte Fluoreszenz-in-situ-Hybridisierung erbrachte den Nachweis eines Bruches des C-MYC-Gens in einer Split-Sonde (ein normales Allel mit fusioniertem (gelb) 5'- und 3'-Anteil der Sonde sowie eines Bruches mit getrenntem grün-rotem Signalpaar), was auf eine Translokation im C-MYC-Gen hinweist. Diagnose: Burkitt-Lymphom

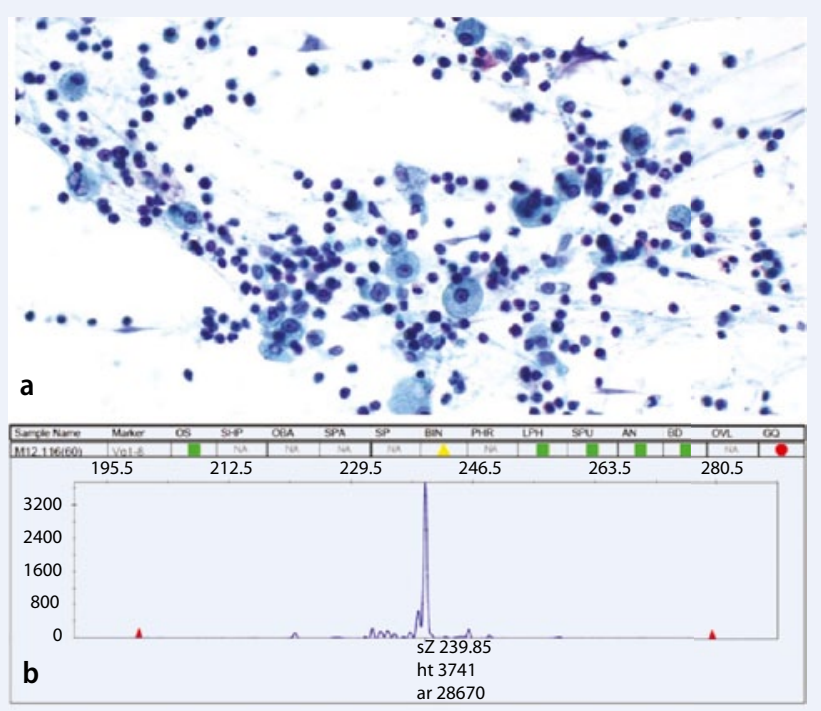

Abb. $8 \Delta$ Ein 55-jähriger Mann mit der Anamnese einer Prolymphozytenleukämie vom T-Zell-Typ, die mit einer Stammzelltransplantation behandelt wurde, entwickelte unklare Lungeninfiltrate. a Die bronchioloalveoläre Lavage erbrachte eine deutliche Vermehrung von reifen Lymphozyten (über $70 \%$ des Zellgehaltes). b Die Klonalitätsanalyse der Restflüssigkeit zeigte eine klonale Rearrangierung des TcRy, übereinstimmend mit dem aus den bioptischen Vorbefunden bekannten pathologischen Klon der Grunderkrankung

\section{Integrative Befundung}

Die Befunderstellung umfasst abschließend eine Beschreibung und Bewertung der Ergebnisse der Ultraschalluntersuchung (Größe, Topographie, Echogenität und Morphologie) sowie der morphologischen, immunphänotypischen (FACS und/oder Immunhistochemie) wie molekularen Veränderungen.

An der Befunderstellung sind mindestens zwei Fachpathologen (Zytologe/in; Hämatopathologe/in mit Zusatztitel für Molekularpathologie) und je nach Untersuchungstechnik ein/e Molekularpathologe/in beteiligt. Bei nicht konklusiven Fällen wird eine Exzisionsbiopsie empfohlen. Analoges Vorgehen wird selbstverständlich bei adäquaten Proben der in anderen Kliniken gewonnenen Punktate [24] bzw. Körper- oder Spülflüssigkeiten angewandt. 


\section{Einschränkungen und Grenzen}

Um die Vorzüge der Methode voll zur Geltung bringen zu können, muss man die Grenzen der zytologischen Diagnostik lymphoproliferativer Erkrankungen kennen.

Grundvoraussetzungen der optimalen Diagnostik sind qualitativ hochstehendes, entnahmeartefaktarmes bzw.- freies zytologisches Material und die Verfügbarkeit von personellen und technischen Kapazitäten für die Durchführung und Interpretation moderner $\mathrm{Zu}$ satzuntersuchungen. In Zentren ohne schwerpunktmäßig ausgebildete Zytologen (diese Weiterbildungsmöglichkeit besteht formell in Deutschland nicht) sind diese Bedingungen häufig nicht erfüllt: Entweder stimmt die Qualität der zytologischen Probe nicht oder das Potenzial einer guten Probe kann nicht optimal für die integrative Diagnostik ausgeschöpft werden. In solchen Fällen ist es empfehlenswert, die fortgeschrittene hämatopathologische Diagnostik primär in Exzisaten vorzunehmen, wie es von Fachgesellschaften für Pathologie empfohlen ist. Leider führt das Unterlassen einer vorgängigen FNP bei dieser Vorgehensweise regelmäßig zu unnötigen Komplikationen und vermeidbaren Fehlern, wie z. B. bei Inzisionsbiopsien von Speicheldrüsenneoplasien. Eine Tendenz zu vermehrter Weiterbildung von Zytopathologen zeichnet sich in der Schweiz seit einigen Jahren $\mathrm{ab}$ und wird auch zunehmend in Deutschland angestrebt.

Die Gradierung der Lymphome kann an zytologischen Proben gelegentlich schwierig sein, wenn auch grundsätzlich in den meisten Fällen eine Unterscheidung von „low grade” (FL-Grad 1 und FL-Grad 2) und „high grade” (Grad 3) des follikulären Lymphoms vorgenommen werden kann [25]. Therapierelevante Feinabstimmungen sind hier häufig nicht möglich und werden im Bericht standardmäßig mit der Empfehlung einer Exzisionsbiopsie vermerkt,.

Eine definitive zytomorphologische Diagnose ist schwierig bzw. definitionsgemäß nicht möglich bei Läsionen, die sowohl durch die Identifizierung der Tumorzellen wie auch deren Umgebung definiert sind. Sehr gute Beispiele stellen sicher die klassischen wie auch die lymphozytendominierten Hodgkin-Lymphome bzw. die T-Zell-Lymphome dar. Hier kann die Diagnose durchaus zytologisch vorgeschlagen werden, jedoch wird eine Biopsie empfohlen, die meist auch von Studienprotokollen verlangt wird. In Fällen von $\mathrm{T} /$ histiozytenreichen großzelligen B-Zell-Lymphomen oder immunsuppressionsassoziierten Lymphoproliferationen wie PTLD, erlaubt eine FNP i.d.R. lediglich eine erste Orientierung zum Ausschluss eines bakteriellen Infektes bzw einer Melanom/Karzinom-Metastase.

Eine FNP liefert im Vergleich zu einer Lymphknotenexzision naturgemäß geringere Mengen an Zellen/Gewebe für allfällige Forschungsprojekte. Dennoch ist es durchaus möglich, frische Zellsuspensate (in Stickstoff gefroren und aufbewahrt) und routinemäßig gefärbte Direktausstriche und/oder Zellblöcke für immunphänotypische und molekulare Analysen auch noch nach Jahren zu verwenden [20, 22].

\section{Fazit für die Praxis}

- Die ultraschallgesteuerte Feinnadelpunktion erlaubt eine optimale Nutzung des gewonnenen Materials bei minimaler Patientenbelastung.

- Das 3-Säulen-Modell der zytologischen hämatopathologischen Diagnostik führt dank der gezielten Triagierung des gewonnenen Materials durch den Zytopathologen zu präzisen, definitiven Diagnosen.

- Durchflusszytometrie an zytologischen Proben erlaubt die Unterscheidung von reaktiven und malignen Prozessen bei reifzelliger Lymphozytose sowie die Klassifizierung reifzelliger B-Zell-Non-Hodgkin-Lymphome gemäß WHO-Kriterien.

- Eine Kombination aus Morphologie, Immunphänotypisierung und molekularpathologischen Untersuchungen ermöglicht eine integrative hämatopathologische Diagnosestellung an zytologischen Proben.

- Wichtig für eine hohe Diagnosesicherheit und optimale Anwendung der Methoden ist eine Beteiligung der Hämatopathologen und Molekularbiologen.

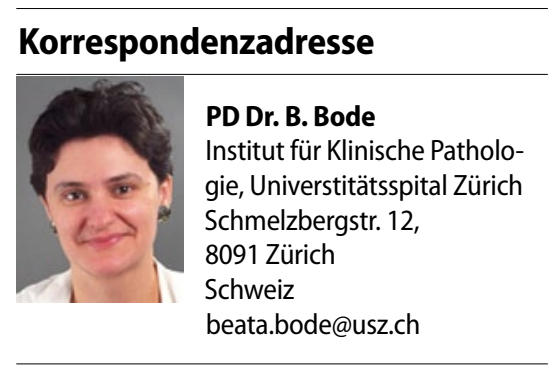

Danksagung. Die Autoren bedanken sich bei Prof. D. Zimmermann und seinem Team sowie J. Roth für die Durchführung der molekulargenetischen Analysen.

Interessenkonflikt. Die korrespondierende Autorin gibt für sich und ihre Koautoren an, dass kein Interessenkonflikt besteht.

\section{Literatur}

1. Bangerter M, Brudler $\mathrm{O}$, Heinrich B et al (2007) Fine needle aspiration cytology and flow cytometry in the diagnosis and subclassification of non-Hodgkin's lymphoma based on the World Health Organization classification. Acta Cytol 51:390-398

2. Barrena S, Almeida J, Del Carmen Garcia-Macias M et al (2011) Flow cytometry immunophenotyping of fine-needle aspiration specimens: utility in the diagnosis and classification of non-Hodgkin lymphomas. Histopathology 58:906-918

3. Bentz JS, Rowe LR, Anderson SR et al (2004) Rapid detection of the $t(11 ; 14)$ translocation in mantle cell lymphoma by interphase fluorescence in situ hybridization on archival cytopathologic material. Cancer Cytopathology 102:124-131

4. Caraway NP (2005) Strategies to diagnose lymphoproliferative disorders by fine-needle aspiration by using ancillary studies. Cancer 105:432-442

5. Caraway NP, Gu J, Lin P et al (2005) The utility of interphase fluorescence in situ hybridization for the detection of the translocation $\mathrm{t}(11 ; 14)$ (q13;q32) in the diagnosis of mantle cell lymphoma on fineneedle aspiration specimens. Cancer 105:110-118

6. Caraway NP, Thomas E, Khanna A et al (2008) Chromosomal abnormalities detected by multicolor fluorescence in situ hybridization in fine-needle aspirates from patients with small lymphocytic lymphoma are useful for predicting survival. Cancer 114:315-322

7. Craig FE, Foon KA (2008) Flow cytometric immunophenotyping for hematologic neoplasms. Blood 111:3941-3967

8. Demurtas A, Accinelli G, Pacchioni D et al (2010) Utility of flow cytometry immunophenotyping in fine-needle aspirate cytologic diagnosis of nonHodgkin lymphoma: a series of 252 cases and review of the literature. Appl Immunohistochem Mol Morphol 18:311-322

9. Dong HY, Harris NL, Preffer Fl et al (2001) Fineneedle aspiration biopsy in the diagnosis and classification of primary and recurrent lymphoma: a retrospective analysis of the utility of cytomorphology and flow cytometry. Mod Pathol 14:472-481

10. Gong Y, Caraway N, Gu J et al (2003) Evaluation of interphase fluorescence in situ hybridization for the $t(14 ; 18)(q 32 ; q 21)$ translocation in the diagnosis of follicular lymphoma on fine-needle aspirates. Cancer Cytopathology 99:385-393 
11. Jorgensen JL (2005) State of the Art Symposium: flow cytometry in the diagnosis of lymphoproliferative disorders by fine-needle aspiration. Cancer 105:443-451

12. Katz RL (2005) Modern approach to lymphoma diagnosis by fine-needle aspiration: restoring respect to a valuable procedure. Cancer 105:429431

13. Lieu D (2008) Cytopathologist-performed ultrasound-guided fine-needle aspiration and coreneedle biopsy: a prospective study of 500 consecutive cases. Diagn Cytopathol 36:317-324

14. Mourad WA, Tulbah A, Shoukri M et al (2003) Primary diagnosis and REAL/WHO classification of non-Hodgkin's lymphoma by fine-needle aspiration: cytomorphologic and immunophenotypic approach. Diagnostic Cytopathology 28:191-195

15. Nicol TL, Silberman M, Rosenthal DL et al (2000) The accuracy of combined cytopathologic and flow cytometric analysis of fine-needle aspirates of lymph nodes. Am J Clin Pathol 114:18-28

16. Richmond J, Bryant R, Trotman W et al (2006) FISH detection of $\mathrm{t}(14 ; 18)$ in follicular lymphoma on $\mathrm{Pa}$ panicolaou-stained archival cytology slides. Cancer 108:198-204

17. Saboorian MH, Ashfaq R (2001) The use of fine needle aspiration biopsy in the evaluation of lymphadenopathy. Semin Diagn Pathol 18:110-123

18. Safley AM, Buckley PJ, Creager AJ et al (2004) The value of fluorescence in situ hybridization and polymerase chain reaction in the diagnosis of b-cell non-hodgkin lymphoma by fine-needle aspiration. Arch Pathol Lab Med 128:1395-1403

19. Schmid S, Tinguely M, Cione P et al (2011) Flow cytometry as an accurate tool to complement fine needle aspiration cytology in the diagnosis of low grade malignant lymphomas. Cytopathology 22:397-406

20. Schmitz R, Renne C, Rosenquist R et al (2005) Insights into the multistep transformation process of lymphomas: IgH-associated translocations and tumor suppressor gene mutations in clonally related composite Hodgkin's and non-Hodgkin's lymphomas. Leukemia 19:1452-1458

21. Swerdlow SH, Campo E, Harris NL et al (Hrsg) (2008) WHO Classification of Tumours of Haematopoietic and Lymphoid Tissues. IARC, Lyon

22. Tinguely M, Rosenquist R, Sundstrom C et al (2003) Analysis of a clonally related mantle cell and Hodgkin lymphoma indicates Epstein-Barr virus infection of a Hodgkin/Reed-Sternberg cell precursor in a germinal center. Am J Surg Pathol 27:1483-1488

23. Wu M (2011) A comparative study of 200 head and neck FNAs performed by a cytopathologist with versus without ultrasound guidance: evidence for improved diagnostic value with ultrasound guidance. Diagn Cytopathol 39:743-751

24. Yasuda I, Goto N, Tsurumi H et al (2011) Endoscopic ultrasound-guided fine needle aspiration biopsy for diagnosis of lymphoproliferative disorders: feasibility of immunohistological, flow cytometric, and cytogenetic assessments. Am J Gastroenterol 107(3):397-404

25. Young NA (2006) Grading follicular lymphoma on fine-needle aspiration specimens-a practical approach. Cancer 108:1-9

26. Zeppa P, Marino G, Troncone G et al (2004) Fineneedle cytology and flow cytometry immunophenotyping and subclassification of non-Hodgkin lymphoma: a critical review of 307 cases with technical suggestions. Cancer 102:55-65
27. Zeppa P, Vigliar E, Cozzolino I et al (2010) Fine needle aspiration cytology and flow cytometry immunophenotyping of non-Hodgkin lymphoma: can we do better? Cytopathology 21:300-310

28. Zhang S, Abreo F, Lowery-Nordberg M et al (2010) The role of fluorescence in situ hybridization and polymerase chain reaction in the diagnosis and classification of lymphoproliferative disorders on fine-needle aspiration. Cancer Cytopathology 118:105-112

\section{Weitere Entstehungs- mechanismen der Leukämie aufgeklärt}

Sowohl bei der Akuten als auch bei der Chronischen Myeloischen Leukämie (AML/ $\mathrm{CML}$ ) führt die vermehrte Produktion reaktiver Sauerstoffspezies (ROS) in den Tumorzellen zu einem Fortschreiten der Erkrankung. Die Ergebnisse zweier aktueller Studien sprechen dafür, dass die Verminderung der ROS-Produktion ein neues therapeutisches Prinzip bei myeloischen Leukämien sein könnte. In Zellen der AML führen große Mengen an ROS-Molekülen zur Inaktivierung eines wachstumsregulierenden Tumorsuppressors. Wird die ROSProduktion experimentell unterbrochen, führt die Reaktivierung dieses Enzyms zu verlangsamtem Wachstum.

CML ist behandelbar durch spezifische Hemmung eines Onkoproteins, resultierend in Apoptose der Krebszellen. In Mitochondrien und Stammzellen werden aber weiterhin verstärkt DNA-schädigende ROS gebildet. Dadurch entstehende Mutationen am Onkogen können die Leukämiezellen wirkstoffresistent werden lassen.

Literatur:

Godfrey R et al (2012) Cell transformation by FLT3 ITD in acute myeloid leukemia involves oxidative inactivation of the tumor suppressor protein-tyrosine phosphatase DEP-1/PTPRJ. Blood 119:4499-4511

Nieborowska-Skorska M et al (2012) Rac2mitochondrial respiratory chain complex III-generated ROS cause genomic instability in chronic myeloid leukemia stem cells and primitive progenitors. Blood 119:4253-4263

Quelle: Universitätsklinikum Jena, www.med.uni-jena.de 